\title{
Perceptual integrality of major chord components
}

\author{
BARBARA E. ACKER and RICHARD E. PASTORE \\ State University of New York, Binghamton, New York
}

\begin{abstract}
In the present study, an accuracy, rather than a reaction time, version of the Garner paradigm was used to evaluate the integrality or separability of major chord components. Tuned (prototype, or P) and mistuned (nonprototype, or NP) sets of root position C-major triads were constructed by holding the $\mathrm{C}$ constant in all stimuli and varying the $\mathrm{E}$ and $\mathrm{G}$ frequencies in 2- and $4-\mathrm{Hz}$ steps. The $\mathrm{P}$ stimuli represent small systematic mistunings in the $\mathrm{E}$ and $\mathrm{G}$ notes relative to an equal-tempered $\mathrm{C}$-major chord. The NP stimuli represent an equivalent range of frequency variation, but relative to a significantly out-of-tune C-major triad. In different experimental sessions, a same-different (AX) task was used to separately evaluate discrimination performance for the $\mathrm{E}$ and $\mathrm{G}$ frequencies as a function of whether the nontarget frequency ( $G$ or $E$ ) was fixed or varied in either a correlated or an orthogonal fashion (with the $\mathrm{C}$ frequency always held constant). Compared with a fixed baseline condition where only the target frequency changed, both chord components exhibited a significant redundancy gain in the correlated conditions and, to varying degrees, significant interference effects in the orthogonal condition, indicating that the chord components function largely in an integral fashion. Relative to the discrimination of $\mathrm{G}$, discrimination of the $\mathrm{E}$ frequency was less influenced by variation in the nontarget $(G)$ frequency, showing that attention, to some degree, could be selectively allocated to the $\mathrm{E}$ chord component. In addition, the results were consistent with previous findings that the functional prototype for the major chord category seems to act as a perceptual anchor, rather than as a magnet, and appears to be located in the physiologically defined area of just temperament, as opposed to the more experientially defined area of equal temperament.
\end{abstract}

Historically, classes of speech and, to a lesser extent, music stimuli were often investigated from the perspective of absolute conceptualizations of categorical perception. ${ }^{1}$ With the central assumption that perceptual categories were discrete (with only irrelevant variation within categories), studies tended to focus on the relationship between category boundary locations and heightened discriminability across the category boundary, with discriminability both expected and found to be reduced within categories. This early work is consistent with discrete categorization processes based on feature detectors, where perceptual differentiation is defined primarily in terms of the limits of feature activation, and thus, the category boundary. In contrast to early discrete feature-detector mechanisms, more recent concepts of categorization have been based on the use of prototypes or exemplars located within categories, thus shifting the focus of importance from the category boundary to the nature of perception within categories. Early research based directly or indirectly on discrete concepts of categorical perception could tell us very little about the nature of internal category structure.

In keeping with the change in theoretical focus, more recent speech studies have featured several alternative

This research was supported by Grant F496209310033 and F490693 10327 from the Air Force Office of Scientific Research. The opinions expressed are those of the authors and do not necessarily represent those of the granting agency. Correspondence should be addressed to B. E. Acker, Psychoacoustics and Auditory Cognition Laboratory, Department of Psychology, State University of New York, Binghamton, NY 13902-6000 (e-mail: bb31074@bingvmb.bitnet). methods to examine the internal structure of speech categories. These studies have begun to provide empirical evidence about the perceptual role of prototypes or exemplars in speech categories (Kuhl, 1991; Li \& Pastore, 1992; Samuel, 1982; Volaitis \& Miller, 1992). Acker, Pastore, and Hall (1995) provided an important musical analog to Kuhl's (1991) investigation of vowel prototypes (described below). The results of the musical category study also provided some new and important insights into the nature of prototypes for musical triads. Building upon these results, the present study adopts some methods from general cognitive science and work on speech prototypes (or exemplars) to more thoroughly investigate the internal structure of a major chord category.

\section{Speech Research}

Kuhl (1991) used a goodness rating task and a discrimination task to evaluate the internal structure of the /i/ vowel category. Sets of high-goodness (prototype, or $\mathrm{P}$ ) and low-goodness (nonprototype, or NP) stimuli were constructed by systematically varying the $F 1$ and $F 2$ frequencies of an $/ \mathbf{i} /$ vowel. Category goodness ratings demonstrated that stimuli within the category are qualitatively graded in terms of distance from a central prototype or modal exemplar, with the $P$ stimuli rated significantly higher than the NP stimuli, and with goodness ratings for $P$ stimuli varying systematically as a function of physical distance (or difference) from the prototype.

With the highest goodness rating consistently applied to a single stimulus central to the $P$ stimulus set, evidence was provided for a prototype located within the category. 
This highest rated stimulus was used as a standard in a subsequent task evaluating discrimination between it and adjacent stimuli in the prototype stimulus set. A lowrated stimulus was used as the discrimination standard for the NP stimulus set. For equal distances from the standard, discrimination was found to be lower for $P$ than for NP stimuli. This finding was interpreted as reflecting reduced discrimination in the region of the prototype, with the prototype acting as a "perceptual magnet" that reduces perceptual distance around it.

\section{Music Research}

Acker et al. (1995) examined the function of prototypes in a major triad category in a manner that paralleled the approach taken by Kuhl (1991; Iverson \& Kuhl, 1995) with the vowel category. In the present study, limited subsets of stimuli from Acker et al. were used; Figure 1 summarizes the current stimulus sets and also illustrates stimulus concepts important in the earlier studies.

In the Acker et al. (1995) study, major chord stimuli for the $P$ set were constructed by varying the frequencies of the $E$ and $G$ (intervals of a 3rd and 5th) of a root position $\mathrm{C}$-major chord by $2-, 4-, 6-$, and $8-\mathrm{Hz}$ steps relative to the standard of an equal-tempered $\mathrm{C}$-major triad; in Figure 1, the 2- and 4- $\mathrm{Hz}$ steps (used in the present study) are the upper-left stimuli centered on $\mathrm{P}$. The $\mathrm{P}$ stimulus represents the theoretical prototype based on equal temperament. Chords for the NP stimulus set (lower-

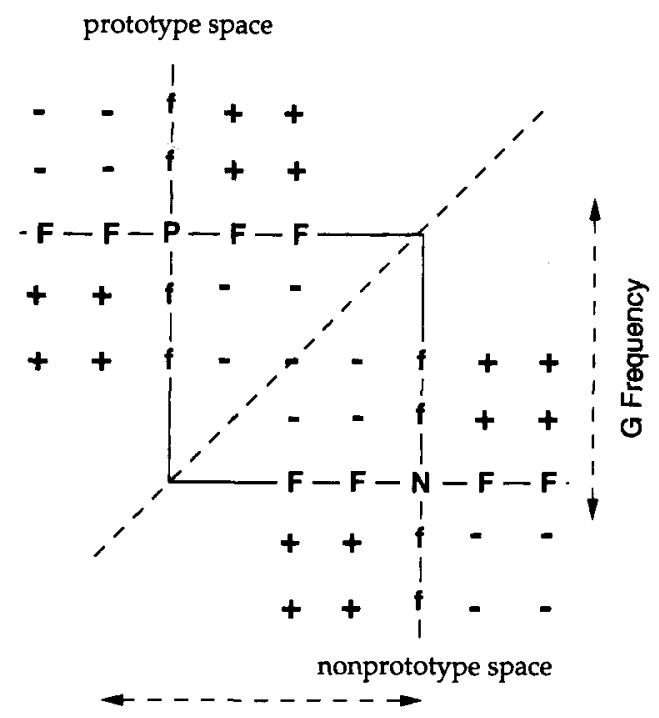

E Frequency

P: Prototype stand. F: Fixed, E dimension
N: Nonprototype stand.+: Positively correlated
$\begin{array}{ll}\text { f: fixed, G dimension } & -: \text { Negatively correlated }\end{array}$

Figure 1. Major chord stimulus space. See text for specifics on stimulus construction. This figure also illustrates the stimuli used in the fixed, correlated, and orthogonal conditions. right stimuli centered on $\mathrm{N}$ in Figure 1) were constructed in a similar fashion, but were based on a mistuned triad (Stimulus $\mathrm{N}$ in Figure 1).

Subjects rated the stimuli for goodness as major chords. As in Kuhl (1991), the estimated prototype was the highest rated stimulus in the $\mathrm{P}$ set, but evaluated for individual musicians instead of a group average. Also similar to the Kuhl vowel results, the NP music stimuli were generally rated significantly lower than were the $\mathbf{P}$ stimuli. These results demonstrate that various members of a major chord category clearly differ in how well they represent the category. There also were some unexpected results. Stimuli that were slightly flat for the $E$ and sharp for the $\mathrm{G}$ (approximating the just-tempered triad) received higher ratings than did the theoretical prototype, reflecting a functional prototype other than the equaltempered triad. The distribution of goodness rating results from this music study further suggested that the individual frequency components of the chord may differentially affect perception of tuning, with the ratings being more systematic as a function of $E$ than $G$ (even after correcting for the difference between the functional and theoretical prototypes). The NP stimuli represented significantly out-of-tune triads, and a systematic decline in ratings, such as that found with the prototype-centered $P$ stimuli, were neither expected nor fc' $ı$ d.

Subjects in the music study also completed a 2IFC discrimination task, where the highest rated stimulus (the functional prototype) was used as a discrimination standard for the P set, and a low-rated stimulus was used as the discrimination standard for the NP set. (Because the individual prototypes were used as the discrimination standards for the predetermined set of stimuli centered on the equal-tempered standard, stimuli were never symmetric around the discrimination standards. Although not relevant for the earlier study, one consequence of this asymmetry was that discrimination was never evaluated for changes in $\mathrm{E}$ independent of $\mathrm{G}$.)

Results, in contrast to Kuhl's (1991) vowel findings, revealed higher discrimination performance for the $P$ stimulus set than for the NP stimulus set. These musical results are consistent with various aspects of musical performance. Specifically, musicians generally neither hear nor play significantly out-of-tune chords, and only small deviations from in-tune chords (i.e., those found in the P context) can be tolerated before the degree of dissonance becomes unacceptable. Therefore, musicians are exposed much more often to the stimuli near the prototype and are trained to be sensitive to the fine-tuning distinctions necessary for accurate performances.

The discrimination results from the Acker et al. (1995) study demonstrated that it is relatively easy to detect changes in overall tuning of chords relative to an in-tune (P) standard, and less so relative to an out-of-tune (NP) standard. These results support an anchor, rather than a magnet, metaphor for the function of a major chord prototype. ${ }^{2}$ While the contrast between a prototype acting as an anchor for the music stimuli and a magnet for the 
speech stimuli is of significant interest and theoretical importance, it is not a factor in the present study.

Although tangential to the goals of the earlier research, the Acker et al. (1995) findings indicated that the individual notes within a major chord may make differential contributions to the tuning of chords. Unfortunately, the asymmetry of the stimulus set prevented a post hoc evaluation of discrimination of the separate chord components. In the present study, potential differences, and possible interactions, between notes in the recognition of the source of the mistuning (E or $G$ ) of Cmajor chords are investigated by having subjects discriminate one specific chord component in the context of different types of variation in the other chord component. As in our previous study, we also investigate differences between $P$ and NP sets of stimuli, here in terms of the integrality or separability of notes. With the $\mathrm{P}$ stimuli, discrimination might be facilitated by an internal representation of a prototypical (or modal exemplar) major chord, whereas discrimination with the NP stimuli might not benefit from (or might be hindered by) a stable internal representation.

\section{Integral and Separable Dimensions}

Current concepts of integral and separable dimensions have been derived largely from the work of Garner (1976), who used discrimination and classification tasks. Two dimensions are said to be separable if attention can be independently allocated to the individual dimensions. In contrast, dimensions are said to be integral if attention cannot be selectively allocated to them. Here, these types of perceptual relationships were evaluated using several experimental conditions in which responding was targeted on one stimulus dimension.

A fixed condition, where only the target dimension changes, was used as a baseline for evaluating performance in separate correlated (both dimensions varying together) and orthogonal (random, independent variation in the nontarget dimension) conditions. Garner (1976) typically assessed reaction time (RT) measures; in comparison with RTs from the fixed condition, a separable relationship is said to exist when there is no significant change in RT across the conditions. Such findings indicate that attention can be selectively and independently allocated to the separate dimensions. If dimensions are integral, there should be a significant improvement in speed (reduction in RT) in the correlated condition, where the sum of the two physical dimensions results in an increased change in the single (integrated) perceptual dimension. Furthermore, interference effects should occur in the orthogonal condition, with RT being slower compared with only the target dimension being varied (fixed condition). The latter finding indicates that selective attention to the separate dimensions is not possible, since perception of the target dimension is affected by change on the other dimension. ${ }^{3}$

The RT analyses described by Garner (1976) require high and approximately equal levels of performance across conditions in order to accurately characterize processing. Equivalent concepts have been described by Tanner (1956) and Ashby and Townsend (1986), but with results analyzed in terms of accuracy. For example, in an orthogonal condition, accuracy should decrease significantly for integral dimensions and should exhibit a significant improvement (redundancy gain) in correlated conditions. Because pilot work for this study indicated that high accuracy was not maintained in all conditions, we decided that RT measures would not adequately capture dimensional processing. Therefore, in the present study we used accuracy measures to evaluate the integral or separable nature of chord components for the $\mathrm{P}$ and NP sets of stimuli. ${ }^{4}$

\section{Predictions}

From a music theory standpoint, it could be hypothesized that the 3 rd interval of a triad should receive more attention than the 5 th interval because the 3 rd determines the major or minor character of the triad. However, results from probe-tone experiments on musical keys provide a basis for an alternative prediction, especially for musically trained subjects. In the probe-tone technique (see, e.g., Krumhansl \& Kessler, 1982), the first 7 notes of a musical scale are presented in sequence, followed by a probe that is randomly selected from one of the 12 notes of the chromatic scale, with subjects asked to rate how well the probe tone completes the 7-note scale. A significantly higher rating is consistently given to the 5th-scale degree than to the 3rd-scale degree. This result demonstrates that, at least in a scale context, the 5th appears to be a stronger influence on the key.

It is well known that many quantitative descriptions of perceptual qualities often can be generalized across research using individual tones, chords, and scales as stimuli. For example, the probe-tone technique has also been used in a chordal context (Krumhansl \& Kessler, 1982), and results were nearly equivalent to those found in a scale context. These consistent results, regardless of tonal context, seem to justify the prediction for the chord context of the present study that the 5th should be more important than the $3 \mathrm{rd}$, and therefore less affected by changes in the $3 \mathrm{rd}$, thus reflecting a higher level of separability. Plomp and Levelt (1965) provided additional support for the importance of the 5th scale interval. In that study, subjects rated intervals that systematically varied in degree of consonance, with results showing that the octave and fifth were most sensitive to deviations from ideal tuning.

Finally, it is possible that the spectral position of chord components, and not the specific frequencies, may be important in the perception of chord tuning. Crowder (1985) has shown that the perception of major chords differs according to their inversions, ${ }^{5}$ with first inversion chords perceived as more major than root position chords. Other research (e.g., Palmer \& Holleran, 1994) has shown that, for musical passages, single changes in the spectrally peripheral notes are easier to detect than are 
those in spectrally central notes. In the present study, we used root position C-major triads, where the $\mathrm{E}$ frequency is embedded between the $\mathrm{C}$ and $\mathrm{G}$ frequencies. It thus is possible that the $\mathrm{G}$ could show a higher level of separability by virtue of its spectrally peripheral location, rather than by its musical role as the dominant in the C-major scale. However, the issue of importance of spectral position becomes relevant only if there is a differential contribution of frequency components to chord perception.

In the preceding discussion, it is assumed that musically trained subjects can listen analytically and thus can (separably) focus on one frequency and ignore information from the other frequency. The ability to encode musical passages by specific frequency would probably require subjects to have absolute pitch, which is a rare ability. In contrast to such absolute coding, several areas of perception research have shown that musicians may listen to stimuli in a more synthetic (or integral) fashion and may most often encode musical passages using interval information (see, e.g., Dowling \& Bartlett, 1981); the use of relative information contained in intervals eliminates the need for absolute pitch identification. For example, in discrimination tasks, a melody is most often considered same even when transposed to a different pitch level compared with a standard melody. If subjects encode chords using relative interval information, such as the relationship between the 3rd and 5th intervals, an integral relationship could be predicted for the component notes, where the relative information from both frequencies is necessary (but possibly not equal) to detect chord mistunings on the basis of the musical intervals.

\section{GENERAL METHOD}

\section{Subjects}

It is assumed that experience plays an important role in the development of the ability to distinguish musical categories. It is often found that subjects lacking musical experience exhibit poor and highly variable performance in tasks using musical stimuli (see, e.g., Crowder, 1985; Plomp \& Levelt, 1965; Shepard, 1964). In addition, preliminary results from ongoing work in our laboratory have shown large differences in the nature of category structure for musically trained and untrained subjects (Acker \& Pastore, 1995). Since the nature of the discrimination task in the present study involves making fine tuning distinctions, musically experienced subjects from the SUNY Binghamton community were used. Our criteria for musical experience were at least 5 years of formal training as well as present involvement in an ensemble. Five of the 6 subjects had completed an undergraduate music degree, or were in the process of completing a music degree, and thus had a background in music theory and history. The 1 subject who was not a music major had completed a music history course, but had no formal training in music theory; the results from this subject were consistent with those from the other 5 subjects. Each of the 6 subjects participated in both experiments, which were run in six 2 -h sessions. Subjects were paid $\$ 5$ per hour for their participation, and all reported normal hearing.

\section{Stimuli}

Two sets of stimuli ( $\mathrm{P}$ and NP) were constructed by generating individual sine tones (12-bit, $10-\mathrm{kHz}$ sample rate), then digitally mixing them to form triads based on a root position $\mathrm{C}$-major triad. Harmonically rich instrument tones with appropriate attack and decay functions might have produced stimuli that were more ecologically valid. However, it would be difficult to vary the fundamental in precise $2-\mathrm{Hz}$ steps for such stimuli. The complete set of stimuli is summarized in Figure 1 , with the codes $(F, f,+,-)$ indicating conditions described below in the Procedure section. The $E$ frequency is mapped along the abscissa and the $G$ frequency along the ordinate.

The P set of stimuli are above the dashed diagonal line in Figure 1 and represent chords that are only slightly mistuned. The theoretical prototype stimulus ( $\mathrm{P}$ in Figure 1 ) was a $\mathrm{C}$-major triad based on equal temperament $(\mathrm{C}=262 \mathrm{~Hz}, \mathrm{E}=330 \mathrm{~Hz}, \mathrm{G}=$ $392 \mathrm{~Hz}$ ), which is the most commonly used tuning system. Twenty-four other stimuli in this context were generated by holding the $\mathrm{C}$ frequency constant and varying the $\mathrm{E}$, the $\mathrm{G}$, or both frequencies by 2 or $4 \mathrm{~Hz}$ in sharp (increasing) and flat (decreasing) directions. Designation of specific stimuli were coded in terms of relative frequency for the $\mathrm{E}$ and $\mathrm{G}$ (e.g., $\mathrm{E}+2 / \mathrm{G}+4$ was the stimulus containing a $2-\mathrm{Hz}$ sharp $\mathrm{E}$ frequency and a $4-\mathrm{Hz}$ sharp $\mathrm{G}$ frequency relative to the $P$ discrimination standard). For 4 of the stimuli, the $G$ was held constant (fixed) and only the $E$ varied ( $F$ stimuli in Figure 1). For another 4 stimuli, the $E$ was held constant (fixed) and only the G varied ( $\mathrm{f}$ stimuli in Figure 1). Sixteen other triads were constructed by varying the $E$ and $G$ in both sharp and flat directions (both the + and - stimuli in Figure 1). Stimuli in the NP context (below the dashed diagonal line in Figure 1) were created in the same way, but were based on a mistuned triad ( $\mathrm{N}$ in Figure 1; $\mathrm{C}=262, \mathrm{E}=338 \mathrm{~Hz}, \mathrm{G}=384 \mathrm{~Hz}$ ). These stimuli were also specified in terms of frequency changes in $\mathrm{E}$ and $\mathrm{G}$, but relative to stimulus $\mathrm{N}$. Each context contained 24 unique stimuli, with 1 stimulus common to both sets ( - stimulus on the dashed line).

Because of the limited frequency range, equal changes in frequency provided a close approximation to equal changes in psychophysical distance. Therefore, the cents scale was not needed to equate perceptual distance. All stimuli were $1,000 \mathrm{msec}$ in duration, with a 50 -msec linear onset and the offset occurring at a positive waveform zero crossing. The stimuli, selected for presentation in a random order, were low-pass filtered at $1 \mathrm{kHz}$ and presented binaurally over TDH-49 earphones at $78 \mathrm{~dB}(\mathrm{~A})$. All subjects were run in commercial sound chambers.

\section{Procedure}

A same-different (AX) task was used to separately evaluate discrimination performance for the $E$ and $G$ frequencies. The task of the subjects was to determine whether or not the $\mathrm{X}$ stimulus was identical in target frequency ( $E$ or $G$ ). Figure 1 summarizes the stimuli in each experimental condition. In all conditions, the standard (P or $N$ in Figure 1) was presented as the first stimulus (A) of each trial. The status of the other (nontarget) variable frequency ( $G$ or $E$ ) defined the nature of (thus label for) the different conditions. For the fixed and correlated conditions, the $\mathrm{X}$ stimulus was the identical standard on same trials and, for different trials, was randomly drawn from the stimulus pool appropriate to the given condition. For the fixed (F) condition, the stimulus pool differed only along the target frequency, with the nontarget frequency held constant (fixed). (In Figure 1, F and $\mathrm{f}$ indicate the different $\mathrm{X}$ stimuli for the fixed $E$ and $G$ discrimination, respectively, with different sets of stimuli defined relative to the $P$ and $N$ standards.) Fixedcondition performance provides a baseline against which changes in performance under the other conditions can be evaluated.

The correlated conditions were used to evaluate the ability of subjects to use information that is redundant across the two frequencies being investigated. Thus, both the $E$ and $G$ frequencies of the standard and comparison stimuli should be identical on same trials and both should differ on different trials. Although typically pooled in many investigations of dimensional relationships, we defined two types of correlated conditions. In the positively correlated condition $(\mathrm{C}+)$, the nontarget frequency varied in the same direction as the target frequency (both frequencies change in 
a sharp or a flat direction). In Figure 1, the + designates the comparison stimuli for the $\mathrm{C}+$ condition on different trials. In the negatively correlated condition $(\mathrm{C}-$ ), the two frequencies varied in opposite directions (one sharp, the other flat); the - symbols in Figure 1 indicate comparison stimuli for the $\mathrm{C}$ - condition on different trials. In both $\mathrm{C}+$ and $\mathrm{C}$ - conditions, the comparison stimulus on same trials was the discrimination standard (either $\mathrm{P}$ or $\mathrm{N}$ in Figure 1).

The orthogonal conditions were used to evaluate the ability of subjects to ignore information on the nontarget frequency, therefore performing the task based only on the presence or absence of a difference between the standard and comparison stimuli along the target frequency. Thus, if $\mathrm{E}$ is the target frequency, the relationship between the standard and comparison stimuli along the nontarget $G$ frequency must be independent of whether the trial was same or different for the E frequency. Thus, the orthogonal conditions are in contrast to the correlated conditions, where the presence or absence of change in one frequency was always accompanied by the (correlated) presence or absence of change in the other frequency.

In the present study, a single discrimination standard for all conditions applied to each of the two (P and NP) stimulus sets. As a result, the independence across the $E$ and $G$ frequencies required by the orthogonal condition was created by having the comparison stimulus differ from the standard along the nontarget frequency on both same and different trials. Thus, in the different trials in the orthogonal condition, all of the stimuli in both the $\mathrm{C}+$ and $\mathrm{C}-$ conditions were used. However, in contrast to the fixed and the two correlated conditions, the standard stimulus ( $P$ or $N$ ) on same trials was followed by an alternative standard that contained the identical target frequency, but differed on the nontarget frequency. With this modification in the definition of same trials, information on the irrelevant frequency could be ignored (and thus have no effect) or it would hinder discrimination of the target frequency. In Figure 1, the alternative standards are designated by the symbol "f" for discrimination based on the $E$ frequency and "F" for discrimination based on the $G$ frequency. (Note that in Figure 1 the alternative standard stimuli [on same trials] for one target dimension [E or G] under the orthogonal condition are [as described above] the comparison stimuli [on different trials] for the other target dimension [G or E] under the fixed condition.)

Each stimulus set $(\mathrm{P}, \mathrm{NP})$ was evaluated in separate experiments. Within each experiment the four conditions (fixed, positively correlated, negatively correlated, and orthogonal) were run for both target frequencies ( $E$ and $G$ ), all in separate blocks of trials. In both experiments, the fixed condition was always run first to help teach the subjects to focus on the specific target frequency. The correlated and orthogonal conditions were counterbalanced across subjects (since no order effects were observed, order was ignored in the subsequent analyses). Subjects were given a detailed description of the conditions, and thus were aware of the frequency relationships in all of the conditions. Subjects were told to focus on the target frequency $(E$ or $G$ ) and to indicate with a buttonpress if the two triads on each trial contained same or different target frequencies. At the conclusion of each trial, visual feedback indicated the correct answer.

\section{Data Analyses and Figures}

All of the data are presented in figures (e.g., Figure 2) that are structured to reflect the organization of the physical stimuli (e.g., Figure 1). One form of statistical evaluation of the results can be accomplished by visually comparing the mean and standard error results across individual stimuli shown in the data figures. The standard error bars of the stimuli varying in both $E$ and $G$ that do not overlap with the error bars for the equivalent fixed stimuli (varying by the same amount only in $E$ or $G$ ) are strong indications of a significant change in discrimination (see Loftus, 1993). However, this type of statistical evaluation does not provide the type of evaluation required by the major goals of the present research.

The first operational goal of the present research was to evaluate discrimination of changes in the $E$ or $G$ frequencies of a root

\section{E Discrimination}

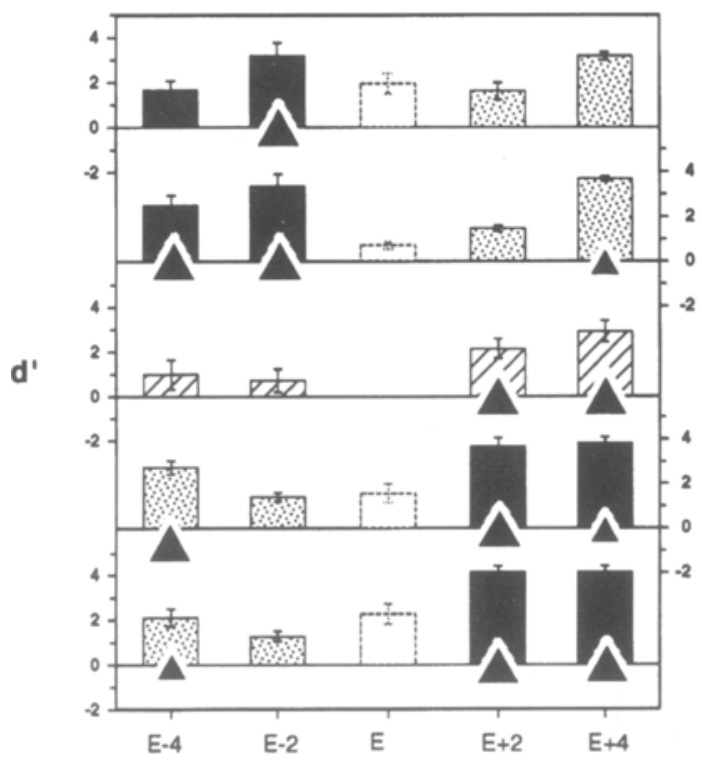

G Discrimination

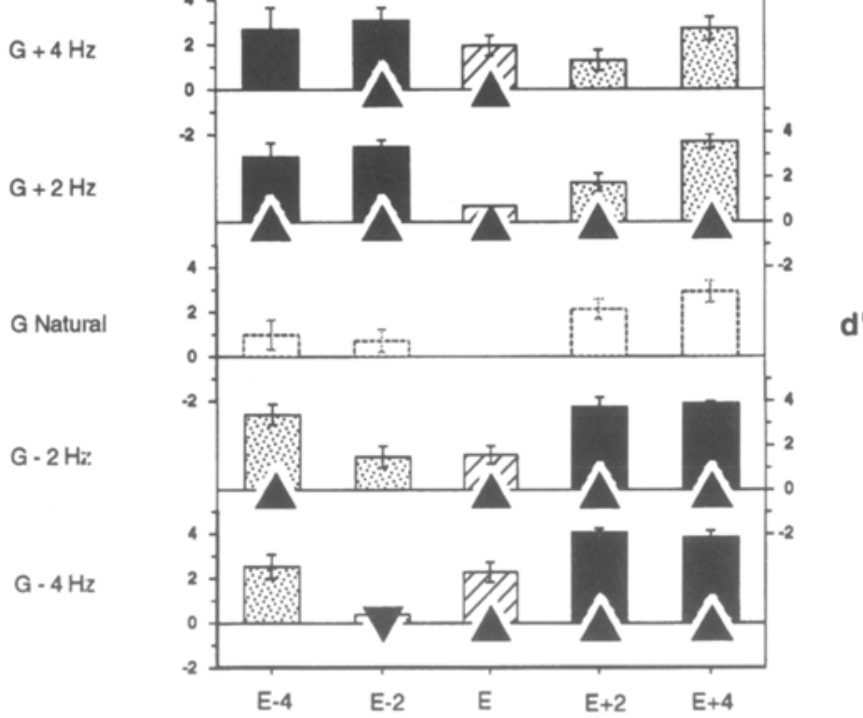

Relative frequency of $\mathrm{E}$ d'

Figure 2. The $d^{\prime}$ values for fixed (striped bars), negatively correlated (dark bars), and positively correlated (dotted bars) discrimination of $E$ (left panel) and $G$ (right panel) for the $P$ stimulus set. Large upward-pointing triangles indicate either significance relative to chance (fixed conditions) or highly significant redundancy gains (correlated conditions); smaller triangles indicate nearly significant gains. Downward-pointing triangles indicate significant decrements. 
position C-major chord when only the given frequency was allowed to vary (fixed conditions). The second set of operational goals was to evaluate possible redundancy gains or interference effects in discrimination performance for correlated and orthogonal changes in the $\mathrm{E}$ and $\mathrm{G}$ frequencies. The descriptive analyses of the results could most easily be (and thus were) accomplished in terms of $d^{\prime}$ values (averaged across subjects) for each stimulus under each condition, as described above. However, in keeping with the second set of goals, the evaluative statistics needed to focus on the within-subject changes in performance under the correlated and orthogonal conditions relative to the performance baseline provided by the fixed condition. Thus it was not appropriate for the primary statistical tests for significance to be based on the variability of performance across subjects under each condition; rather, they needed to be based on the variability across subjects of the (within-subject) changes in performance under the correlated and orthogonal conditions relative to the fixed condition. For the present research, the options of available statistical tests were limited by several factors. For example, one does not expect equivalent performance for both 2 - and $4-\mathrm{Hz}$ changes, and one thus cannot simply expect homogeneity of variance across the different stimuli utilized in the current investigation. In addition, the experimental design required us to compare performance for a number of different stimuli with baseline performance provided by a single fixed condition. Most important, we did not focus on average performance under different conditions or on a detailed analysis of performance for each individual stimulus summarized in Figure 1 under every possible condition. Instead, our goals required the identification of overall trends in the incidence of redundancy gains or interference effects (thus, differences in performance within individual subjects) under the different stimulus conditions.

Two different sets of statistical tests were conducted. The Dunnett $t$ test (Winer, 1962), which compares each of multiple conditions with a single set of control or baseline results, provides a probability correction for multiple tests. Although otherwise appropriate, the error term for this (usually two-tailed) test is based on variability in absolute performance across subjects within conditions (pooled across conditions), rather than variability in change of performance. Although Dunnett $t$ tests were performed on the results, reports of significance are based on the Student $t$ test performed on the differences in $d^{\prime}$ for individual subjects across the conditions being compared. Since there are specific predictions of direction of change, all of the latter tests are one-tailed with a criterion of .05 (significant findings are designated by large, filled triangles in data figures). Since our interest was in identifying trends in interference or redundancy gain, discrimination of stimuli approaching significance $(.05<p<1.0)$ is also reported (small, filled triangles in data figures). The estimates of significance from the one-tailed Student and the two-tailed Dunnett $t$ tests exhibited a very high degree of correspondence, with the few differences primarily involving a change between significance and "approaching significance."

The fixed-condition $t$ tests evaluate whether discrimination is better than chance $\left(d^{\prime}=0\right)$. In the correlated and orthogonal conditions, $t$ tests used within-subject comparisons to test for a significant difference between performance with a given stimulus and performance in the comparable fixed condition (i.e., identical change in target frequency). Since the nontarget frequency in the correlated conditions either should be ignored or should produce a redundancy gain (improved performance), the one-tailed statistic tests for improved performance relative to the comparable fixed condition (significance indicated by an upward-pointing triangle). Since the nontarget frequency in the orthogonal condition should either be ignored or should produce interference effects (a decrement in performance), the one-tailed statistic evaluates a reduction in performance relative to the comparable fixed condition (significance indicated by a downward-pointing triangle)

\section{EXPERIMENT 1 Prototype Discrimination}

In Experiment 1, discrimination for the prototype set of stimuli was investigated. Pilot work had seemed to indicate that with these relatively in-tune chords, discrimination for changes in $\mathrm{E}$ was easier than in $\mathrm{G}$. In an effort to insure that subjects understood the various tasks, all conditions were first run using the presumed easier $\mathrm{E}$ as the target dimension for discrimination. ${ }^{6}$

\section{Results and Discussion}

Discrimination results in the fixed and correlated (not orthogonal) conditions for the $\mathrm{E}$ and $\mathrm{G}$, plotted as average values of $d^{\prime}$ (along with standard error bars), are shown respectively in the left and right panels of Figure 2. The organization of each of the two panels of Figure 2 parallels that of the prototype stimuli in Figure 1, with $E$ varying along the abscissa and $G$ along the ordinate. In the left panel, the fixed-condition results for $E$ (striped bars on the vertically centered horizontal axis, corresponding to the F stimuli in Figure 1) serve as a baseline for comparison with the correlated E discrimination condition results. (These same results also are shown, for comparison, along with the results for $\mathrm{G}$ discrimination [unpatterned bars in right panel]. Likewise, the fixed condition results for the G [bars on the horizontally centered vertical axis, corresponding to $f$ stimuli in Figure 1] are also shown in both panels, but with significance indicated only in the right panel [G results]. These same fixedcondition results for $E$ and $G$ are also shown with the orthogonal results in Figure 3, again without indications of significance for the fixed condition.)

\section{Fixed Discrimination of $\mathbf{E}$}

In the fixed condition, discrimination of neither the $-2 \mathrm{~Hz}$ nor the $-4 \mathrm{~Hz}$ change in $\mathrm{E}$ was significantly greater than chance. The average $d^{\prime}$ value for stimulus $\mathrm{E}-2$ was somewhat below the typical signal detection criterion of $d^{\prime}=1$ for threshold. However, stimulus E-4 had an average $d^{\prime}$ value of 1.0 and did approach statistical significance. In contrast to the reduced sensitivity to changes in the flat direction, sensitivity was high for +2 and $+4 \mathrm{~Hz}$ changes to $\mathrm{E}$, with the results for both stimuli being significantly greater than chance. If subjects were basing their judgments only on the E component, these results would indicate that sensitivity is somewhat less for small changes in a flat direction than for small changes in a sharp direction. If, however, interval information is important, then the decreased performance as $E$ changes in a flat direction could reflect an improved tuning of these chords relative to the equal-tempered discrimination standard (see General Discussion).

\section{Correlated Discrimination of $\mathbf{E}$}

The negatively correlated $(\mathrm{C}-$ ) condition (opposite direction of changes for $E$ and $G$ ) results are shown as solid bars in Figure 2. There was a significant redundancy gain (improved discrimination performance rela- 
E Discrimination

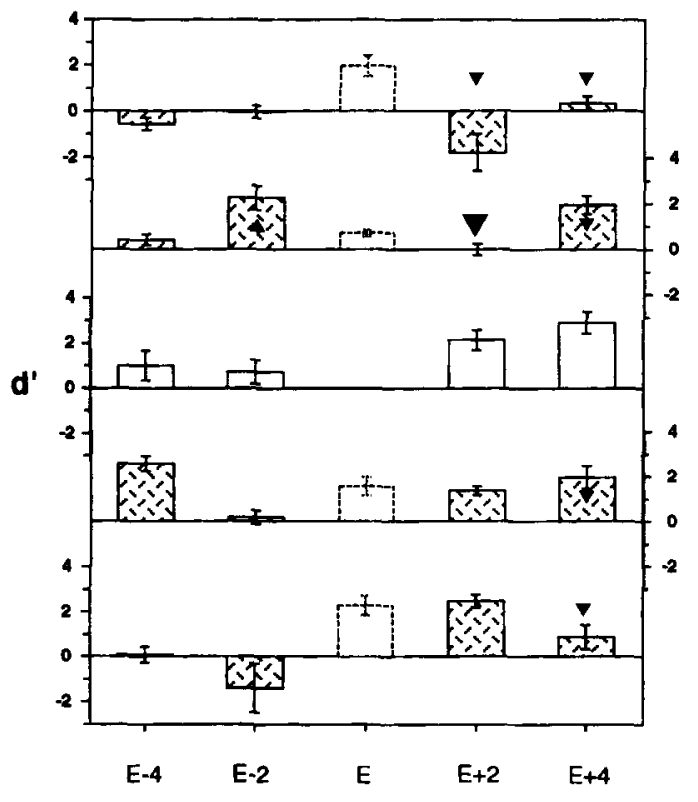

G Discrimination

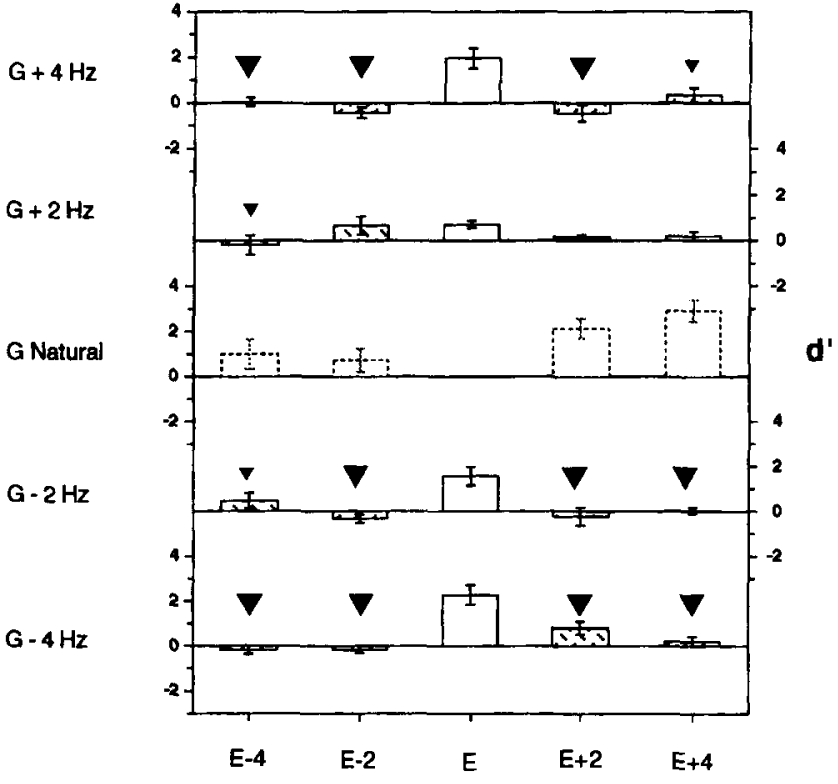

Relative frequency of $E$

Figure 3. Orthogonal discrimination of $E$ (left panel) and $G$ (right panel) for the $P$ stimulus set. Large downward-pointing triangles indicate significant decrements; smaller triangles indicate nearly significant decrements.

tive to the fixed condition) for 6 out of the 8 stimuli, and the results approached significance for a 7 th stimulus (small filled triangle). These $\mathrm{C}$ - results appear to indicate that changes in the target dimension are easily detected when the nontarget dimension changes in the opposite direction.

A very different pattern of results was found for the positively correlated $(\mathrm{C}+)$ condition (dotted bars in Figure 2). With $\mathrm{C}+$, the redundancy gain was significant for only one stimulus $(E-4 / G-2)$ and approached significance for only 2 other stimuli $(E-4 / G-4$ and $E+4 /$ $\mathrm{G}+2$ ). These results indicate that discrimination of component changes in the same direction $(\mathrm{C}+)$ is difficult. Therefore, the significant redundancy gains for prototype $\mathrm{E}$ discrimination are seen for most of the $\mathrm{C}$ - stimuli and very few of the $\mathrm{C}+$ stimuli.

\section{Fixed Discrimination of $G$}

The $\mathrm{G}$ results for fixed and correlated conditions are summarized in the right panel of Figure 2. All fixed G stimuli (striped bars on the vertical axis) were discriminated at significantly greater than chance. However, unlike discrimination for $\mathrm{E}$, discrimination for $\mathrm{G}$ did not exhibit a difference based on the direction of change (sharp or flat).

In terms of the fixed-condition results across the target dimensions ( $E$ and $G$ ) for equally flat stimuli, there was higher performance for the $\mathrm{G}$, although these differences were not significant. A finding of better discrimination for the $G$ frequency would be consistent with previously discussed results indicating that the 5thscale degree exerts somewhat more influence in a scale context (Krumhansl \& Kessler, 1982) and is more sensitive to tuning deviations (Plomp \& Levelt, 1965). Alternatively, the peripheral location of the $G$ in the chord could have aided discrimination (Palmer \& Holleran, 1994). However, as noted above, the fixed-condition discrimination for slightly flat $\mathrm{E}$ stimuli probably reflects the influence of the just-tempered prototype, which, compared with the equal-tempered chord ( $\mathrm{P}$ standard), has a flat $E$ and a slightly sharp G. Averaged across all fixed stimuli, the baseline performance, against which redundancy gain and interference effects are measured, was equivalent for $\mathrm{E}\left(d^{\prime}=1.69\right)$ and $\mathrm{G}\left(d^{\prime}=1.63\right)$ discrimination.

\section{Correlated Discrimination of $\mathbf{G}$}

$\mathrm{G}$ discrimination under the $\mathrm{C}$ - condition (dark bars) exhibited a significant redundancy gain (relative to fixed G) for 7 out of the 8 stimuli. For the one exception (stimulus $E-4 / G+4$ ), most subjects exhibited a redundancy gain, but 2 subjects exhibited performance decrements for both E and G discrimination. Acker et al. (1995) had indicated considerable variability in location of the functional prototype; functional prototypes for these 2 subjects, although not directly evaluated, may have been more flat for $E$ and sharp for $G$ than a just-tempered chord.

In contrast to the $\mathrm{C}$ - results, when $\mathrm{E}$ and $\mathrm{G}$ are positively correlated, significant redundancy gains are found only for 3 of the 8 stimuli. One of these stimuli, E-4/ 
G-2, also exhibited a redundancy gain for correlated discrimination based on $\mathrm{E}$, thus possibly indicating something unusual about this stimulus relative to both the fixed $\mathrm{E}$ and $\mathrm{G}$ stimuli. Another $\mathrm{C}+$ stimulus, $\mathrm{E}-2 / \mathrm{G}-4$, exhibited a significant decrement relative to fixed $\mathrm{G}$. With this stimulus, performance was close to chance for all subjects.

In terms of the correlated condition results across $\mathrm{E}$ and $\mathrm{G}$ discrimination, more stimuli under $\mathrm{G}$ discrimination were altered by correlated variation of the other frequency (10 with redundancy gain and 1 with a decrement) than under $E$ discrimination ( 7 with redundancy gain). For discrimination of both frequencies, significant redundancy gains were seen for far more stimuli under the $\mathrm{C}-(6+7=13$ stimuli $)$ than under the $\mathrm{C}+(1+$ $3=4$ stimuli) conditions.

\section{Orthogonal Discrimination of $\mathbf{E}$}

The results for $\mathrm{E}$ discrimination in the orthogonal condition are shown in Figure 3 (left panel, patterned bars). In general, performance in the orthogonal condition was relatively poor. When $E$ and $G$ both changed in a sharp direction, there were decrements in performance relative to fixed $\mathrm{E}$. However, with high variability for some stimuli, only 1 of these stimuli $(E+2 / G+2)$ achieved statistical significance based on the within-subject comparisons; the decrements for the 3 other stimuli approached statistical significance. When $\mathrm{E}$ and $\mathrm{G}$ both changed in a flat direction, performance level generally dropped, with 1 stimulus $(E-2 / G-4)$ exhibiting negative values of $d^{\prime}$, indicating that the subjects could discriminate the conditions, but were probably inappropriately attributing perceived changes to $G$ rather than to $E$. However, none of the changes in performance (relative to the fixed baseline) either achieved or approached statistical significance.

When the $E$ and $G$ changed in opposite directions, none of the stimuli exhibited significant within-subject decrements relative to the comparable fixed-condition results. However, 2 of these stimuli $(E+4 / G-2$ and $E+4 /$ $\mathrm{G}-4$ ) exhibited decrements that did approach significance relative to the high performance for fixed $E+4$. Stimulus $E-2 / G+2$, which closely approximated the functional just-tempered prototype, exhibited improved performance (relative to comparable fixed $(E-2)$ condition), which approached statistical significance.

\section{Orthogonal Discrimination of G}

The results for discrimination of $\mathrm{G}$ with orthogonal variation in $E$ are shown in the right panel of Figure 3. Ten of the 16 stimuli exhibited significant decrements in performance relative to fixed $\mathrm{G}$ performance, with the decrements for 3 of the remaining stimuli approaching statistical significance. The 1 stimulus that did not exhibit a decrement in performance $(E-2 / G+2)$ approximated the just-tempered chord. Thus, in contrast to the very few stimuli that exhibited even marginally significant decrements in the orthogonal condition for $\mathrm{E}$ discrimination, most of stimuli in the orthogonal condition for $\mathrm{G}$ discrimination exhibited significant interference effects.

\section{Prototype Discrimination Summary}

Discrimination of both $E$ and $G$ exhibited significant redundancy gains when there was correlated variation in the other frequency. Although to a much lesser degree for E, both also exhibited significant interference when there was orthogonal variation in the other frequency. This pattern of findings is generally consistent with the notion that $\mathrm{E}$ and $\mathrm{G}$ are integral in defining the perception of chords.

Although fixed-condition discrimination for $\mathrm{E}$ and $\mathrm{G}$ exhibited equal average $d^{\prime}$ values, discrimination of $\mathrm{E}$ was less influenced by correlated variation in $G$ and much less influenced by orthogonal variation in $G$. There thus is some evidence that the $\mathrm{E}$ component in the root position C-major chord plays a greater role in perception.

We have also found that the nature of the correlation is important in determining the magnitude of interaction between the $E$ and $G$ components of chords, with the negatively correlated stimuli (upper-left and lower-right quadrants in Figures 2 and 3) exhibiting greater redundancy gain in the correlated condition (Figure 2), and the positively correlated stimuli (at least for $E$ ) tending to exhibit greater interference effects in the orthogonal condition (Figure 3). A possible explanation of the latter findings is provided in the General Discussion.

\section{EXPERIMENT 2 Nonprototype Discrimination}

The integral nature of the $E$ and $G$ in the $P$ context suggests that interval information, and thus the relationship between the component frequencies, is important in the perception of the tuning of a major chord, with attention not able to be allocated solely to one frequency. The $\mathrm{P}$ stimulus set represents only slightly mistuned triads, and intervals of the theoretical prototype could serve as a point of contrast for nearby stimuli, resulting in successful discrimination based on the degree of mistuning. If overall tuning of the standard is important, a different pattern of results should be found with the NP stimulus set, where the NP discrimination standard contains no in-tune intervals. In Experiment 2, the procedures from Experiment 1 and the NP stimulus set were used (see lower-right-hand portion of Figure 1) to evaluate these potential differences.

\section{Results and Discussion}

\section{Fixed Discrimination of $\mathbf{E}$}

The results for E discrimination with the NP stimuli under the fixed and correlated conditions are summarized in the left panel of Figure 4. The overall performance level was lower with the NP stimuli than with the P stimuli, consistent with the results of Acker et al. (1995). ${ }^{7}$

The 2 stimuli with $E$ changing in the flat direction $(E-4$ and $E-2)$ relative to the out-of-tune standard $(N)$ were discriminated at significantly better than chance. 
These 2 stimuli are closest to the prototype (P) stimulus set, and thus are relatively more in-tune than $\mathrm{N}$, the discrimination standard; this relative tuning difference might have provided the basis for the better performance for these stimuli. When the $\mathrm{E}$ changes were in a sharp direction, and thus slightly more out-of-tune than the discrimination standard, discrimination performance was essentially at chance.

\section{Correlated Discrimination of $\mathbf{E}$}

Discrimination of changes in $\mathrm{E}$ when there were correlated changes in $G$ resulted in significant redundancy gains for 4 stimuli, all of which represent negative correlations between $E$ and $G$. Two of these stimuli $(E-4 /$ $\mathrm{G}+4$ and $\mathrm{E}-2 / \mathrm{G}+4$ ) are closest to the prototype stimulus set and thus should be the least dissonant of the nonprototype stimuli. Three other stimuli with negative correlations between $E$ and $G(E+4 / G-4, E+2 / G-2$, and $\mathrm{E}-4 / \mathrm{G}+2$ ) approached statistical significance. Thus, 7 out of the 8 stimuli with negatively correlated changes in $\mathrm{E}$ and $\mathrm{G}$ exhibited redundancy gains that either achieved or approached statistical significance. None of the $\mathrm{C}+$ stimuli were discriminated at a level significantly different from the comparable fixed stimuli.

\section{Fixed Discrimination of G}

The right panel of Figure 4 summarizes the fixed and correlated $\mathrm{G}$ discrimination results with the NP stimuli.
As found with $E$ as the target dimension, overall performance was much lower than with the $P$ stimuli, consistent with findings from the earlier Acker et al. (1995) study. In fact, all of the fixed-condition $G$ stimuli were discriminated at approximately chance, with none (including those closest to the $\mathbf{P}$ stimuli) differing significantly from chance. Thus, in contrast to the equal average results found in the $P$ stimulus set, fixed-condition discrimination for the NP stimuli was much better for $\mathrm{E}$ $\left(d^{\prime}=0.69\right)$ than for $\mathrm{G}\left(d^{\prime}=0.21\right)$, but with both averages below the typical measure of threshold.

\section{Correlated Discrimination of $\mathbf{G}$}

All C - stimuli exhibited a significant redundancy gain. However, only one $\mathrm{C}+$ stimulus $(\mathrm{E}-4 / \mathrm{G}-2)$ exhibited a significant redundancy gain, and stimulus $E+4 / G+2$ approached significance.

\section{Orthogonal Discrimination of $\mathbf{E}$}

The results for E discrimination in the context of orthogonal variation in $\mathrm{G}$ are shown in the left panel of Figure 5. None of the orthogonal stimuli exhibited a significant decrement in performance relative to baseline (fixed E) performance. However, stimulus $\mathrm{E}+4 / \mathrm{G}-2$ exhibited a significant improvement in performance (two-tailed $t$ test) relative to fixed $\mathrm{E}$ discrimination; that is, fixed $\mathrm{E}$ baseline was not significantly different from chance.

\section{E Discrimination}

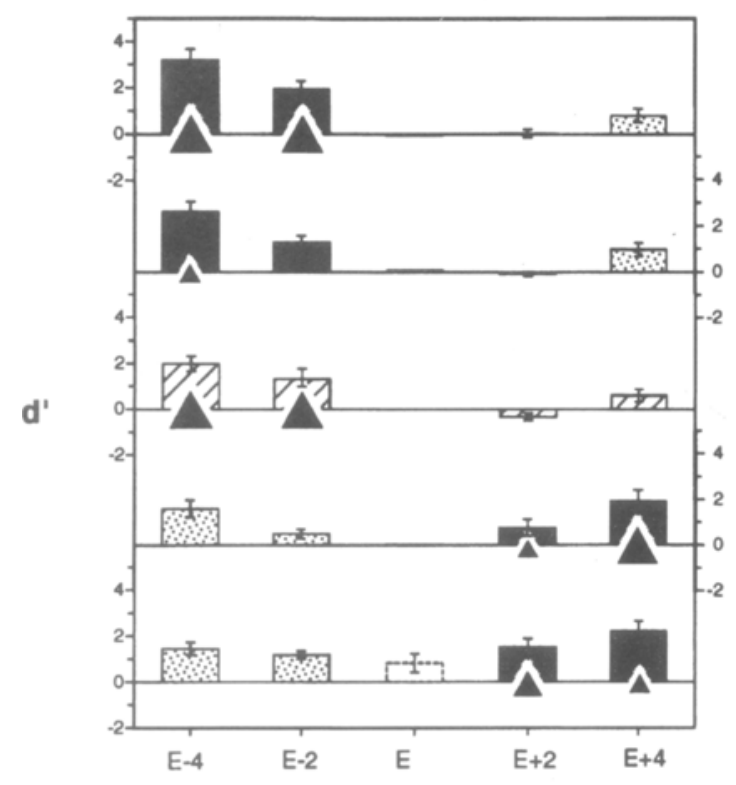

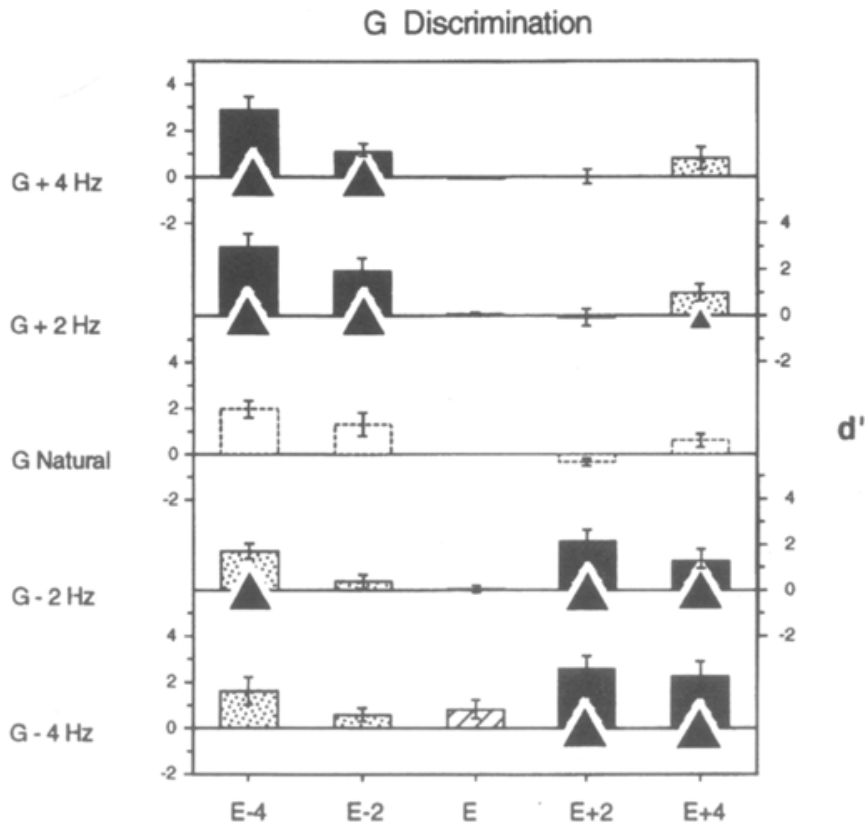

Relative frequency of $E$

Figure 4. The $d^{\prime}$ values for fixed (striped bars), negatively correlated (dark bars), and positively correlated (dotted bars) discrimination of $E$ (left panel) and $G$ (right panel) for the NP stimulus set. Large upward-pointing triangles indicate either significance relative to chance (fixed conditions) or highly significant redundancy gains (correlated conditions); smaller triangles indicate nearly significant gains. 
E Discrimination

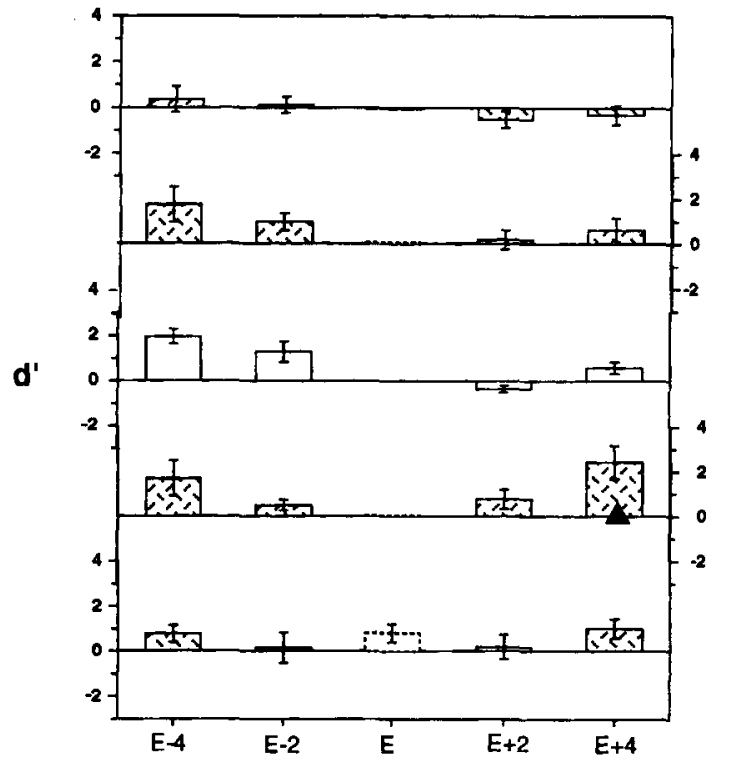

G Discrimination

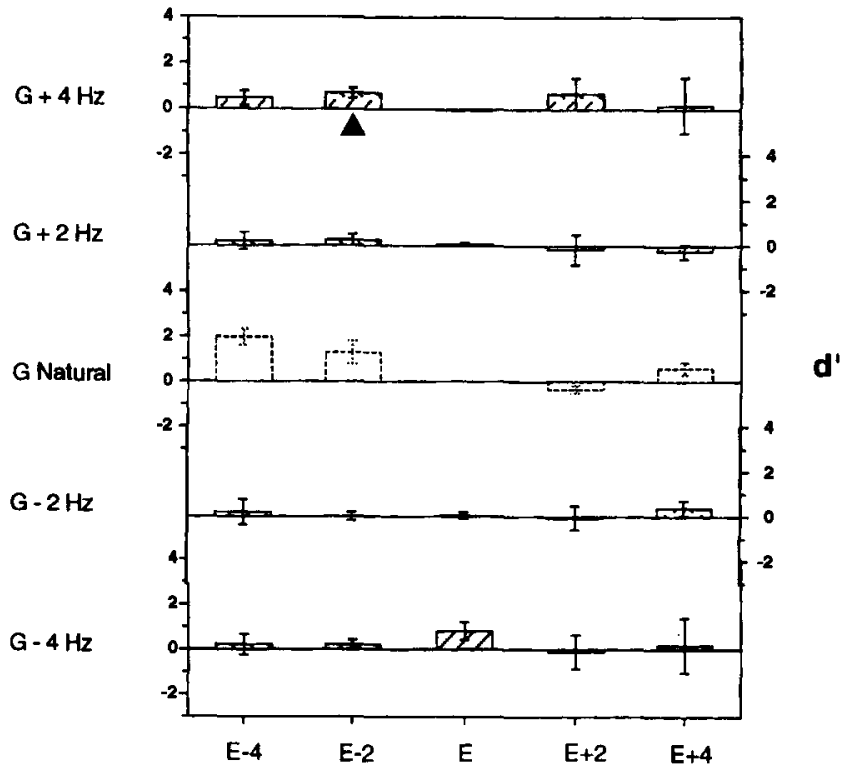

Relative frequency of $E$

Figure 5. Orthogonal discrimination of $E$ (left panel) and $G$ (right panel) for the NP stimulus set.

\section{Orthogonal Discrimination of G}

None of the stimuli exhibited a significant decrement in performance. This finding should not be surprising, since performance is compared with the fixed $G$ discrimination, which was uniformly at chance. One of the stimuli $(E-2 / G+4)$ was discriminated at significantly better than the baseline $\mathrm{G}$ performance. However, the average $d^{\prime}$ value for this stimulus $\left(d^{\prime}=0.81\right)$ was below the typical criterion for threshold.

\section{Nonprototype Discrimination Summary}

The finding of redundancy gains for both E- and Gcorrelated discrimination provides evidence for the generally integral nature of these chord components for the NP stimulus set, but with some qualifications.

In contrast to the $\mathrm{E}$ frequency in the NP context, nearly all of the stimuli in either the correlated or the orthogonal condition for the $G$ target frequency were discriminated at a level that was not significantly above fixed $G$ performance, which, in turn, was discriminated at chance. The lower incidence of redundancy gain for E discrimination may directly reflect the initially higher baseline (fixed condition) performance against which the gain is evaluated. However, both the higher fixed performance for $E$ and the higher incidence of redundancy gain for $G$ discrimination (reflecting the contribution of $\mathrm{E}$ to $\mathrm{G}$ performance) are indicators that the $E$ frequency seems to play a greater role in the perception of root position Cmajor chords. Finally, as was also found with the P stimuli, a redundancy gain was found far more often under the $\mathrm{C}$ - than under the $\mathrm{C}+$ conditions, even after factor- ing out proximity to the $\mathrm{P}$ stimulus set (half of the $\mathrm{C}-$ stimuli [upper-left quadrant of NP space in Figure 1] are closer to the prototype than are all $\mathrm{C}+$ stimuli $[+$ symbols], but the other half [lower-right quadrant] are farther away).

\section{GENERAL DISCUSSION}

Predictions for the present study (see above) were based on some previous empirical findings, as well as some music and auditory theory concepts, which provided a basis for expecting the possibility of a separable relationship between the component notes in a major triad, with each component making a qualitatively different contribution to perception of tuning. Other findings and concepts provided a basis for expecting an integral relationship. The present research, using a version of a paradigm designed specifically to evaluate dimensional relationships, found evidence for the component chord notes to be generally integral, rather than separable. Each target note exhibited significant redundancy gains in the correlated conditions and, for $\mathrm{G}$ discrimination with the prototype stimulus set, substantial interference effects in the orthogonal condition. (The lack of interference effects for orthogonal NP discrimination can be explained by a generally poor ability to discriminate either component, with the fixed-condition results often establishing a null baseline against which to evaluate a performance decrement). Thus, when $E$ (in contrast to $G$ ) is the nontarget frequency, it provides extra information that often serves to facilitate performance in the correlated condi- 
tions, but that cannot be ignored (thus interfering with discrimination) in the orthogonal conditions.

While this summary of findings generally implies an integral relationship between the $\mathrm{E}$ and $\mathrm{G}$ components of a C-major chord, it is also clear that the two frequencies do not contribute equally to discrimination. After addressing the nature of the integral relationship between $G$ and $E$, we return to this finding of asymmetric or differential salience of the $\mathrm{E}$ component in terms of possible importance of the musical versus spectral position of this tone in the chord.

\section{Integral Nature of Components}

The present, general finding of an integral relationship between component notes in a major chord is consistent with research showing the importance of interval relationships in music perception. For example, Dowling (1978) has shown that, in the processing of melodies, human listeners generally encode musical passages by using relative (frequency ratios), not absolute (actual frequency), information. Perception of chord tuning also appears to use relational information, with representations probably stored according to frequency relationships, and not the specific frequency values of individual notes. This finding was seen for both the relatively in-tune $P$ stimuli and the mistuned NP stimuli.

Perception of chords in terms of the component musical intervals provides a basis for understanding the consistent finding that the negatively correlated stimuli exhibited a significantly higher incidence of redundancy gain than did the positively correlated stimuli. When there is an equal absolute change in both components, the positively and negatively correlated stimuli are not equal in the number of intervals that differ from the discrimination standard. In the $\mathrm{C}+$ condition, the two frequencies moved up or down as a unit, resulting in a difference in only two intervals within the chord: $(\mathrm{C}-\mathrm{E})$ and $(\mathrm{C}-\mathrm{G})$. Similarly, in the fixed condition, the modification of one of the three components always resulted in a difference in two intervals: $(C-E)$ and $(E-G)$ when $E$ was the target frequency. However, with the $\mathrm{C}-$ condition, all three intervals always differed across the stimuli being discriminated: $(C-E),(E-G)$, and $(C-G)$. A greater number of modified intervals in the $\mathrm{C}-$ condition could provide more cues, such as greater total (interval) dissonance, to facilitate overall discrimination relative to both the fixed and $\mathrm{C}+$ conditions. This difference in overall magnitude of mistuning is relevant for discrimination of both relatively tuned (P) and mistuned (NP) stimuli.

If this analysis is valid, several implications are suggested. If it is primarily the total amount of mistuning that is critical for discrimination, both the physical component notes and the physical component intervals may be integral in the perception of chords. However, if the number of mistuned intervals is critical for discrimination (or if the tuning of one interval is more important than the total amount of mistuning across all three intervals), it is possible that the component notes may be integral in defining the component intervals, but that the intervals themselves may be at least partially (and, possibly, asymmetrically) separable. For reasons to be developed below, the better discrimination for the $E$ component in the correlated and orthogonal conditions is consistent with the conjecture that the intervals are at least partially separable. An empirical evaluation of a separable (or integral) relationship among the component intervals in a triad would require that experiments like those in the present study be conducted (to the extent possible) with intervals (rather than notes) manipulated in a fixed, correlated, and orthogonal fashion.

\section{Spectral Position Versus Specific Frequency}

The apparent greater importance of the E component in both the $P$ and NP contexts raises the question of whether the $E$ is discriminated more easily than the $G$, or whether the frequency in the middle of the chord is discriminated better (see note 4). Several empirical findings (cited earlier) and music theory principles (e.g., the role of the third in defining the nature of the triad) predict that it is indeed the $E$ that is being discriminated, but there are also other factors that may have contributed to the current findings of a greater importance for the $\mathrm{E}$ to discrimination performance. The root position chords in the present study were created with pure tones (thus, without the higher harmonics important for instrument timbre). Thus, the $E$ is in the center of the chord spectrum and the $\mathrm{G}$ is at the upper spectral extreme. This contrast in spectral position may allow for several basic perceptual differences. One possible basic perceptual difference is upward spread of masking from $\mathrm{C}$ to $\mathrm{E}$, and from both $\mathrm{C}$ and $\mathrm{E}$ to $\mathrm{G}$. Alternatively, if the intervals between notes are at least partially separable, the central $\mathrm{E}$ component in the root position chord is a component in two similar, small interval ratios (C-E is $5: 4 ; E-G$ is $6: 5)$, whereas the $\mathrm{G}$ is a component in a broader range of interval ratios $(\mathrm{C}-\mathrm{G}$ is $3: 2 ; \mathrm{E}-\mathrm{G}$ is $6: 5)$.

Some aspects of the importance of spectral position could be evaluated by utilizing the current paradigm with chord inversions of the current stimuli. If spectral frequency or chroma is important in the discrimination of chord components, the E should maintain its superior level of discrimination regardless of spectral position. If, instead, spectral position or range of interval ratios is more important, if the $\mathrm{G}$ is placed between the $\mathrm{C}$ and $\mathrm{E}$ (a first inversion chord), discrimination of G should improve, and discrimination of the $\mathrm{E}$ should be reduced.

\section{Influence of Tuning System}

The frequency specifications of the prototype standard were based on equal temperament, which is the most commonly used tuning system in Western music. Equal temperament enables transposition on keyboard instruments, but sacrifices pure ratios between the intervals, making the 5ths ( $\mathrm{G}$ in the present study) slightly flat and the 3 rds ( $E$ in the present study) sharp. An alternative, less common tuning system, just temperament, maintains more pure interval ratios that are reflective of basic perceptual and physiological findings (Plomp \& 
Levelt, 1965). Compared with equal temperament used for the triads in both the Acker et al. (1995) and the present study, the $\mathrm{G}$ in just temperament is sharp by approximately $1 \mathrm{~Hz}$ and the $\mathrm{E}$ is flat by approximately $3 \mathrm{~Hz}$.

Early researchers (e.g., Moran \& Pratt, 1926) found that sequential intervals of pure tones were adjusted by musically trained subjects to agree with equal temperament. Therefore, one would expect that the extensive experience of the practiced musicians who served as subjects should have defined the C-major prototype at the frequency values specified by equal temperament. In addition, there is evidence that experience with specific instruments can affect preferred tuning of scales of sawtooth waves (see, e.g., Loosen, 1995). However, primary instrument of study (e.g., violin vs. piano) did not seem to be correlated either with the individual prototypes identified in the Acker et al. (1995) study or with the pattern of discrimination in the present study, both of which used chords composed of simultaneous tones. Furthermore, the goodness ratings from Acker et al. show that individual functional prototypes for chords are in the area defined by just temperament rather than equal temperament.

Because the chord tones were presented simultaneously, a preference for just temperament would be expected because of greater spectral purity, due to the lack of beats (roughness), in these chords. Chords based on equal temperament have frequency ratios that would produce beats. Thus, the discrimination standard used for the $\mathrm{P}$ stimuli in the present study, based on equal temperament, probably did not reflect the functional (just-tempered) major chord prototype. This difference between the functional chord prototype and the discrimination standard provides an explanation for some of the P stimulus discrimination findings.

Recall that in Experiment 1, discrimination for a fixed $E$ was poorer when the $E$ changed in a flat direction relative to the discrimination standard than when changed in a sharp direction. If the integral nature of the chord components results in a discrimination response criterion based on total degree of mistuning, then poorer discrimination for the chords containing slightly flat $\mathrm{E}$ frequencies is logical. When the different stimuli had a slightly flat $E$, and thus were located in the area of the just-tempered prototype, they were probably perceived as somewhat more spectrally pure than was the equal-tempered discrimination standard, and thus should have led to an incorrect same response. Relative to the discrimination standard, chords containing a sharp E frequency represented a greater degree of mistuning, and thus were discriminated more easily, as seen in Figure 2.

A prototype based on just temperament can also account for the slight differences in the fixed-condition performance for the $\mathrm{E}$ and $\mathrm{G}$. Recall that the $\mathrm{G}$ in the equaltempered discrimination standard is flat by only $1 \mathrm{~Hz}$ compared with just temperament, but that the $E$ is sharp by $3 \mathrm{~Hz}$. Therefore, in the actual (equal-tempered) standard for the P stimulus set, the $\mathrm{G}$ frequency component was only slightly different from the functional (just-tempered) prototype, but the $\mathrm{E}$ frequency component was $3 \mathrm{~Hz}$ sharp. The differences in goodness ratings in the Acker et al. (1995) study indicate that subjects are sensitive to differences of $2 \mathrm{~Hz}$. Therefore, a $3-\mathrm{Hz}$ difference in the $\mathrm{E}$ component of the standard should be perceptually significant, while a $1-\mathrm{Hz}$ difference in $\mathrm{G}$ may not be discriminable. Thus, in the present study, the standard stimulus in the fixed $G$ condition was probably not perceptibly different from the just-tempered prototype, whereas all different stimuli were further from the just-tempered prototype, and thus, mistuned (except possibly for stimulus $\mathrm{E} 0 / \mathrm{G}+2$, where discrimination was, in fact, poor). In contrast to the stimuli in the fixed $\mathrm{G}$ condition (e.g., $\mathrm{E} 0 / \mathrm{G}+2$ ), stimuli $\mathrm{E}-2 / \mathrm{G} 0$ and $\mathrm{E}-4 / \mathrm{G} 0$, both nominally different stimuli, are within $1 \mathrm{~Hz}$ of the functional prototype. This clearly differential degree of tuning should have enhanced discrimination for the $G$ fixed stimuli relative to the fixed $\mathrm{E}$ stimuli for equivalent differences relative to the discrimination standard. One important general conclusion from the findings in our present and previous studies is that even extensive experience with equal temperament does not seem to override prototypes based on physiological aspects of the auditory system.

\section{Detection and Identification}

The generally integral nature of the frequencies and the patterns of discrimination for $E$ and $G$ in the $P$ stimulus set seem to indicate an important dissociation between detection and identification. Subjects appeared to be able to detect small mistunings in major chords, but had difficulty identifying the source of the mistuning, especially when the mistuning was associated with the $\mathrm{G}$ component. Specifically, the fixed and correlated conditions of the present research demonstrated that, with the $P$ stimuli, musically trained subjects could readily detect changes in the tuning of major chord components, but did not seem to be consistently able to respond differentially to the separate $E$ and $G$ components. In the orthogonal conditions, the range of variability provides evidence that some listeners produced negative values of $d^{\prime}$ for some stimuli, indicating some ability to discriminate between the stimuli, but also indicating a misattribution of the nature of the difference. This distinction between detection and identification can also be found in other psychoacoustic research. For example, Hirsh (1959; see also Pastore, Harris, \& Kaplan, 1982) found that only $2 \mathrm{msec}$ was needed for subjects to detect an asynchrony in the onset time of two tones, but an onset difference of $20 \mathrm{msec}$ was required to identify which tone occurred first. Similarly, Divenyi and Hirsh (1974) found that subjects could detect the different onsets for a sequence of three tones with component durations of $4 \mathrm{msec}$, but that they required a component duration of $20 \mathrm{msec}$ to identify the order of the tones in the sequence. Thus, it is not unusual to find that listeners can detect a difference that is too small for adequate identification of the source of the difference. With an increase in the mistuning of either or both components, discrimination performance should, and did, improve. 


\section{Extent of Prototype Influence}

In addition to the new results, the current work replicates Acker et al. (1995), where musically trained subjects demonstrated better discrimination within a stimulus set built around a prototype than within a nonprototype stimulus set. The results from the two studies suggest that a category prototype acts as an interior anchor, and that nearby stimuli are not assimilated to the prototype (as in a magnet effect), but are contrasted to it. In the original Acker et al. study, the NP stimulus set was intended to evaluate perception in the absence of strong influence from a prototype. However, because Acker et al. followed the design of the Kuhl (1991) vowel study, one of the stimuli in the NP set was the theoretical (equaltempered) prototype and a number of the stimuli were quite close to the functional prototype. Thus, it is possible that the NP results may have reflected some influence of the prototype. Furthermore, because the previous study employed individual stimulus discrimination standards, which were based on the estimated individual prototypes, the discrimination results could not be evaluated relative to either a consistent standard or a standard that was central to the stimulus set.

In contrast to the previous study, the NP stimuli in the present study did not contain either the theoretical or the functional prototype, with all stimuli discriminably different from both prototypes. Furthermore, discrimination in both the P and NP stimulus sets was relative to a central discrimination standard. As a result, we could evaluate the symmetry, or asymmetry, of results. As discussed above, the asymmetry of fixed-condition results in the P set, while not expected, can be explained in terms of the offset functional prototype relative to the discrimination standard based on the theoretical (equal-tempered) prototype. Factoring out the effects of the offset theoretical prototype, the asymmetry of $\mathrm{C}+$ and $\mathrm{C}-$ condition results for the $\mathrm{P}$ stimuli was unexpected and provided some insights (described above) into the possible basis of perceptual coding for triads. With the NP stimuli, we found an asymmetry of results for correlated discrimination based on both $E$ and $G$, with discrimination highest for stimuli closest to the theoretical and the functional prototype. This last finding further demonstrates that the existence of a nearby prototype in perceptual space (even if not contained in the set of physical stimuli presented) can alter patterns of discrimination, and does so by enhancing performance.

\section{SUMMARY AND CONCLUSION}

The present study clearly validates several important findings from the earlier Acker et al. (1995) study. In contrast to findings reported for vowel prototypes, the prototype for musical triads functions as a perceptual anchor, improving discrimination. Furthermore, despite the often assumed importance of extensive experience with equal temperament, the functional triad prototype for musicians seems to be based on just temperament. The new findings indicate that the component notes in a triad function in a perceptually integral fashion (for both tuned and mistuned chords), probably in defining the interval between the component notes with some aspect of interval tuning playing a role in discrimination, as indicated by differences in the positively and negatively correlated conditions. The present results also indicated that, although the two component frequencies investigated do function in an integral fashion, the spectrally centered $E$ frequency played a more important role in the perception of chord tuning than did the G frequency located at the spectral edge of the chord. Finally, there was some indication that the intervals between notes may not necessarily be perceptually integral, but also that they probably are not fully separable. Thus, conclusions about the perceptual nature of stimulus dimensions need to be described in a manner that is more precise than is a simple, binary statement of their integral or separable nature.

\section{REFERENCES}

ACKer, B. E., \& PAstore, R. E. (1995). The role of experience in the development of category structures [Abstract]. Journal of the Acoustical Society of America, 97, 3241.

Acker, B. E., Pastore, R. E., \& Hall, M. D. (1995). Musical chord discrimination: Perceptual magnet or anchor? Perception \& Psychophysics, 57, 863-874.

AshBY, F. G., \& TownSEND, J. T. (1986). Varieties of perceptual independence. Psychological Review, 93, 154-179.

BURns, E. M., \& WARD, W. I. (1978). Categorical perception-Phenomenon or epiphenomenon: Evidence from experiments in the perception of melodic musical intervals. Journal of the Acoustical Society of America, 63, 456-468.

CROWDER, R. G. (1985). Perception of the major/minor distinction: II. Experimental investigations. Psychomusicology, 5, 3-24.

DiVENYI, P. L., \& HiRSH, I. J. (1974), Identification of temporal order in three-tone sequences. Journal of the Acoustical Society of America, 56, 144-151.

Dowling, W. J. (1978). Scale and contour: Two components of a theory of memory for melodies. Psychological Review, 85, 341-354.

Dowling, W. J., \& BARTLETT, J. C. (1981). The importance of interval information in long-term memory for melodies. Psychomusicology, 1, 30-49

GARNER, W. R. (1976). Interaction of stimulus dimensions in concept and choice processes. Cognitive Psychology, 8, 98-123.

HiRSCH, I. J. (1959). Auditory perception of temporal order. Journal of the Acoustical Society of America, 31, 759-767.

Howard, D., Rosen, S., \& Broad, V. (1992). Major/minor triad identification and discrimination by musically trained and untrained listeners. Music Perception, 2, 205-220.

IVERSON, P., \& KuHL, P. (1995). Mapping the perceptual magnet effect for speech using signal detection theory and multidimensional scaling. Journal of the Acoustical Society of America, 97, 553-562.

Krumhansl, C. L., \& Kessler, E. J. (1982). Tracing the dynamic changes in perceived tonal organization in a spatial representation of musical keys. Psychological Review, 89, 334-368.

KuHL, P. K. (1991), Human adults and human infants show a "perceptual magnet effect" for the prototypes of speech categories, monkeys do not. Perception \& Psychophysics, 50, 93-107.

LI, X., \& PAstore, R. E. (1992). Evaluation of prototypes in perceptual space for a place contrast. In M. E. H. Schouten (Ed.), The auditory processing of speech (pp. 303-308). New York: De Gruyter.

LOCKE, S., \& Kellar, I. (1973). Categorical perception in a nonlinguistic mode. Cortex, 9, 355-369. 
Lofrus, G. R, (1993). Editorial comment. Memory \& Cognition, 21, $1-3$

Logan, G. (1988). Toward an instance theory of automatization. Psychological Review, 95, 492-527.

Loosen, F. (1995). The effect of musical experience on the conception of accurate tuning. Music Perception, 12, 291-306.

MacMillan, N. A., Braida, L. D., \& Goldberg, R. F. (1987). Central and peripheral processes in the perception of speech and nonspeech sounds. In M. E. H. Schouten (Ed.), Psychophysics of speech perception (pp. 28-45). Dordrecht: Martinus Nijhof.

Massaro, D. W. (1987). Categorical partition; A fuzzy-logical model of categorization behavior. In S. Harnad (Ed.), Categorical perception: The groundwork of cognition (pp. 254-283). New York: Cambridge University Press.

Miller, J. L. (1977). Properties of feature detectors for VOT: The voiceless channel of analysis. Journal of the Acoustical Society of America, 62, 641-648.

Moran, H., \& Pratt, C. C. (1926). Variability of judgments on musical intervals. Journal of Experimental Psychology, 9, 492-500.

Palmer, C., \& Holleran, S. (1994). Harmonic, melodic, and frequency height influences on the perception of multivoiced music. Perception \& Psychophysics, 56, 301-312.

PASTORE, R. E., HaRRIS, L. B., \& KAPLAN, J. K. (1982). Temporal order identification: Some parameter dependencies. Journal of the Acoustical Society of America, 71, 430-436.

Pastore, R. E., Schmuckler, M. A., Rosenblum, L., \& Szczesiul, R. (1983). Duplex perception with musical stimuli. Perception \& Psychophysics, 33, 469-474.

Plomp, R., \& LeVelt, J. M. (1965). Tonal consonance and critical bandwidth. Journal of the Acoustical Society of America, 38, 548-560.

Samuel, A. G. (1982). Phonetic prototypes. Perception \& Psychophysics, 31, 307-314.

ShEPARD, R. N. (1964). Circularity in judgments of relative pitch. Journal of the Acoustical Society of America, 36, 2346-2353.

Siegal, J. A., \& Siegal, W. (1977). Categorical perception of tone intervals: Musicians can't tell sharp from flat. Perception \& Psychophysics, 21, 399-407.

Studdert-Kennedy, M., Liberman, A. M., Harris, K.S., \& CoOper, F. S. (1970). Motor theory of speech perception: A reply to Lane's critical review. Psychological Review, 77, 234-249.

TANNER, W. P. (1956). Theory of recognition. Journal of the Acoustical Society of America, 28, 882-888.

Volaitis, L. E., \& Miller, J. L. (1992). Phonetic prototypes: Influence of place of articulation and speaking rate in the internal structure of voicing categories. Journal of the Acoustical Society of America, 92, 723-735.

Winer, B. J. (1962). Statistical principles in experimental design (pp. 89-92). New York: McGraw-Hill.

\section{NOTES}

1. Representative examples of speech research include StuddertKennedy, Liberman, Harris, and Cooper (1970). Representative music research includes Burns and Ward (1978); Locke and Kellar (1973); Siegal and Siegal (1977); Howard, Rosen, and Broad (1992); Pastore, Schmuckler, Rosenblum, and Szczesiul (1983). However, some researchers did consider a graded internal structure of speech categories (e.g., Massaro, 1987; Miller, 1977; Samuel, 1982).

2. In psychophysics, an anchor is associated with heightened discrimination (Macmillan, Braida, \& Goldberg, 1987). The term anchor is thus used here in this traditional manner to contrast with the metaphor of a magnet, which describes reduced discrimination. The distinction between anchor and magnet may reflect the precision of "tuning" of the prototype; the narrowly tuned prototype for fine-tuning of chords could act as an anchor, whereas a prototype for a broad perceptual category (e.g., vowels, and possibly chord type) could act as a magnet.

3. In the original Garner (1976) conceptualization, if dimensions are equally discriminable and perfectly integral, RT should be faster in a correlated condition than in a fixed condition. If dimensions are separable, no RT improvement would be expected in the correlated condition. However, a race horse model of RT (see, e.g., Logan, 1988) would predict slightly better performance in the correlated condition for separable dimensions, because there now are two independent sources of relevant information present. In addition to simple integral and separable notions, two other possible perceptual relationships include configural and asymmetric separable. Configural relationships are said to exist when a dimension is not perceptually separable in the orthogonal conditions, but shows no facilitation in correlated conditions. Asymmetric separable dimensions demonstrate facilitation in correlated conditions, but for only one of the dimensions.

4. It should be noted that the issues of anchor/magnet and integrality/ separability are independent. Our previous research leads us to predict higher overall discrimination performance in the $\mathrm{P}$ context, with the prototype acting as an anchor. However, dimensions could be integral, with the prototype defining overall tuning, but not enabling discrimination of individual components. Different predictions for th $P$ and $\mathrm{NP}$ contexts can still be made, even if components are integral. For example, the level of redundancy gain may be greater in the $\mathbf{P}$ context than in the NP context. Additionally, it is possible that components could be separable in one context and integral in the other context.

5. A root position $\mathrm{C}$-major chord contains the $\mathrm{C}$ as the lowest frequency, followed by the $\mathrm{E}$ and $\mathrm{G}$. A first inversion chord places the $\mathrm{C}$ in the uppermost frequency position, with the $\mathrm{E}$ as the lowest frequency and the $\mathrm{G}$ embedded in the middle.

6. Due to an interaction of software errors that were not discovered until several subjects had completed the conditions, the data from some of the orthogonal conditions were not correctly printed and the actual trial-by-trial data failed to be stored in a disk file. As a consequence, the data for $\mathrm{E}$ discrimination in the prototype context are based on only 3 subjects for the $2-\mathrm{Hz}$ difference and only 2 subjects for the 4- $\mathrm{Hz}$ difference. The conclusion (below) of better discrimination for $\mathrm{E}$ than for $\mathrm{G}$ in the orthogonal, prototype context, condition reflects consistent findings seen in the results for (1) the subjects with complete data for all conditions and (2) the other subjects comparing conditions with complete data.

7. Whereas slightly reduced discrimination for stimuli immediately adjacent to a prototype (relative to more distant stimuli) could be mistaken for a perceptual magnet effect, such a conclusion would mix two different levels of analysis. Standard psychophysics predicts that discrimination ability should increase as a function of increasing difference between the comparison stimuli and the discrimination standard. The current manipulation of the $\mathrm{E}$ and $\mathrm{G}$ frequencies (including their interaction) is varying both physical and perceptual differences between the stimuli being discriminated, and thus provides a type of psychophysical evaluation of such discrimination within the P and NP sets of stimuli. Performance need not be, and was not, the same for equal physical changes within different sets of stimuli. The notion of a magnet or anchor refers to the latter differences in the discrimination of equivalent stimulus differences across two sets of stimuli. The present results are consistent with earlier findings, where discrimination is better relative to a $\mathrm{P}$ than to an NP standard, showing that a prototype acts as an anchor for discrimination of chords.

(Manuscript received March 23, 1995; revision accepted for publication November 15,1995 .) 